\title{
Laser Ablation
}

National Cancer Institute

\section{Source}

National Cancer Institute. Laser Ablation. NCI Thesaurus. Code C111241.

Removal, separation, detachment, extirpation, or eradication of a body part, pathway, or function by light amplification by stimulated emission of radiation. 